Ann. Zootech., I969, 18 (3), 277-298.

\title{
COMPARAISON ENTRE LE PATURAGE ET L'AFFOURAGEMENT EN VERT POUR LA PRODUCTION DE VIANDE BOVINE
}

\author{
M. CHENOST et C. DEMARQuILLY \\ avec la collaboration technique de G. CUYLLE \\ Station de Recherches sur l'Élevage des Ruminants, \\ Centre de Recherches de Clermont-Ferrand, 63-Saint-Genès-Champanelle \\ Institut national de la Recherche agronomique
}

\section{SOMMAIRE}

\begin{abstract}
Pendant 4 années successives, de 1963 à 1966, nous avons comparé au domaine du Pin-auxHaras (Normandie) les vitesses de croissance et d'engraissement de 2 lots comparables de bœufs de 30 mois pâturant des prairics temporaires à base de graminées ou recevant en stabulation de l'herbe de même âge fauchée sur ces prairies. En i 965 et 1966, les animaux d'un troisième lot en stabulation ont reçu $3 \mathrm{~kg}$ d'orge par jour en supplément de l'herbe.

Les bœufs recevant de l'herbe coupée s'engraissent aussi vite et aussi bien que ceux qui pâturent. La croissance moyenne a été respectivement en stabulation et au pâturage de I I $55 \mathrm{~g}$ et I I $26 \mathrm{~g}$ par jour de la mise à l'herbe jusqu'au départ des premiers animaux pour l'abattoir (début scptembre) et de I 039 et I $067 \mathrm{~g} /$ jour pour la totalité de la période d'engraissement. La distribution de $3 \mathrm{~kg}$ d'orge par jour aux animaux augmente relativement peu (de l'ordre de $4^{\circ} \mathrm{g} / \mathrm{jour}$ jusqu'au début septembre et de $\mathrm{r} 7 \mathrm{O} \mathrm{g} / \mathrm{jour}$ pour la totalité de la période d'engraissement) les gains de poids individuels car elle entraîne une diminution équivalente $(3 \mathrm{~kg} / \mathrm{jour}) \mathrm{de}$ la quantité de matière sèche d'herbe ingérée. En revanche, elle diminue significativement de 20 jours environ la duré a de la période d'engraissement.

La quantité de croît produite à l'hectare a été en moyenne de $75 \mathrm{r} \mathrm{kg}$ par hectare fauché contre $672 \mathrm{~kg}$ par hectare pâturé soit une différence de I I,8 p. Ioo. Elle a été accrue de $36 \mathrm{p}$. Ioo par l'apport de $3 \mathrm{~kg}$ d'orge.
\end{abstract}

\section{INTRODUCTION}

L'affouragement en vert des bovins, plus particulièrement des vaches laitières, est une pratique très ancienne qui consiste à apporter de l'herbe verte fauchée chaque jour aux animaux maintenus à l'étable. Appelé par certains zéro-pâturage, ce système d'alimentation a longtemps été limité à certaines étables situées à proximité ou à 1'intérieur des villes (étables de nourrisseurs) mais surtout à certaines régions, l'Alsace. notamment, aux exp^oitations très morcelées, sans clôtures fixes, ou à forte densité rurale, où les cultures prédominaient sur les herbages. Il était souvent saisonnier et 
était surtout de règle pendant les périodes où les prés produisaient peu alors que des fourrages cultivés étaient disponibles ; seigle et navette au tout début du printemps, mais et moha au milieu de l'été, choux en arrière-saison.

Dans sa forme traditionnelle ce système présentait l'inconvénient de demander beaucoup de main-d'œuvre, notamment parce que dans les anciennes étables à stabulation entravée, la distribution des fourrages aux animaux se faisait à la main. Au cours des dernières années, le système a joui en France d'un regain d'intérêt. I,es causes doivent en être recherchées dans l'intensification fourragère qui met à la disposition de l'agriculteur des fourrages très productifs mais dont l'exploitation en pâturage n'est pas toujours facile et dans l'existence de machines (servant à l'ensilage) qui facilitent la fauche, le ramassage et la distribution aux animaux, notamment s'ils sont en stabulation libre. Cette dernière offre en outre l'avantage de ne pas nécessiter l'enlèvement journalier du fumier.

La comparaison entre l'affouragement en vert et le pâturage a fait l'objet d'un certain nombre d'expériences (cf. revues de HUFFMAN, I959; ARNON, I960 ; WATSON et Runcie, I960). En règle générale, l'affouragement en vert permet une meilleure utilisation de l'herbe en réduisant les refus dus aux déjections et au piétinement et permet par là, une augmentation de la production animale à 1'hectare. Cette augmentation est d'autant plus grande que le fourrage employé a un port dressé (ArNon, I960). En revanche, les performances individuelles des animaux en stabulation sont en général plus faibles que celles des animaux en pâturage. Ces expériences ont été réalisées essentiellement avec des vaches laitières ou des animaux en croissance; très peu l'ont été avec des bœufs à l'engrais (cf. revues bibliographiques de HufFmax, I959; ArNoN, I960; WATSON et RUNCIE, I960). Le problème essentiel pour nous était de savoir s'il était possible d'engraisser des bœufs en leur distribuant à l'auge de l'herbe fauchée sur des prairies temporaires à base de graminées, sans apport complémentaire d'aliments concentrés, tel que cela se passe au pâturage.

Pendant 4 années successives nous avons donc mesuré au domaine expérimental du Pin-au-Haras, en Normandie, les vitesses de croissance et d'engraissement de 2 lots comparables de boufs de 30 mois pâturant des prairies temporaires ou recevant en stabulation de 1'herbe de même âge et de même stade fauchée sur ces prairies. Au cours des 2 dernières années nous avons rajouté un deuxième lot en stabulation pour étudier les répercussions d'un apport complémentaire de $3 \mathrm{~kg}$ d'orge par animal et par jour en supplément de l'herbe distribuée à volonté. Les animaux ont été maintenus en expérience jusqu'à ce qu'ils aient atteint un état d'engraissement optimum, ou dans le cas contraire durant toute la période de croissance active de l'herbe.

Nous avons mesuré la digestibilité de l'herbe distribuée et la quantité ingérée à l'auge, le gain de poids et la qualité de la carcasse de chaque animal, les surfaces utilisées et par suite le gain de poids par hectare.

\section{MATÉRIEL ET MÉTHODES}

\section{Animaux et prairies}

Les prairies temporaires utilisées étaient à base d'une seule graminée (Ray-grass anglais ou italien, Fétuque des prés) associée à du Trèfle blanc. La figure I donne les espèces utilisées et les calendriers d'exploitation. Le semis a eu lieu sur le sol nu au printemps ou à l'automne et les 

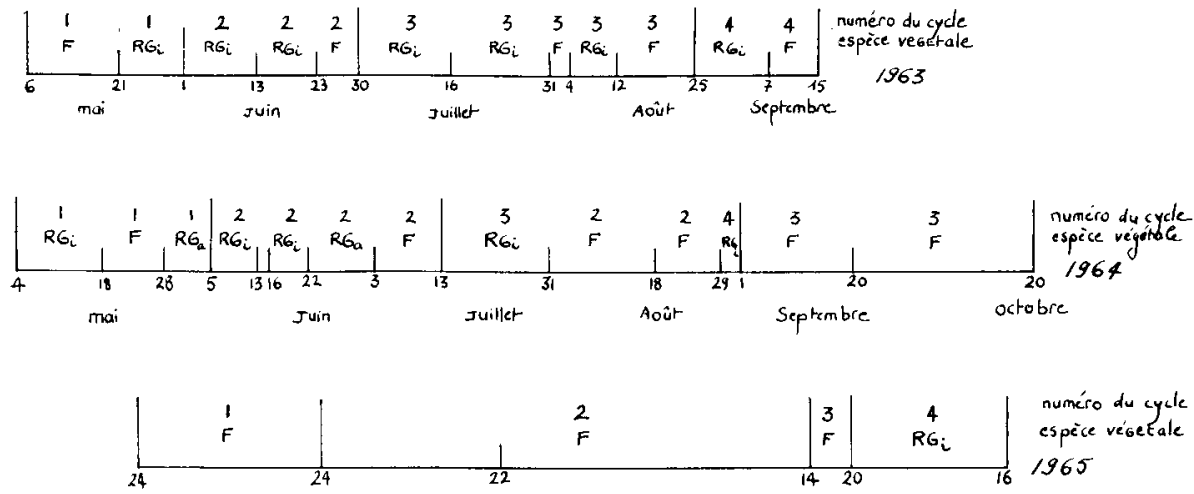

mai Juin Juillet Aoüt Septembre octobre

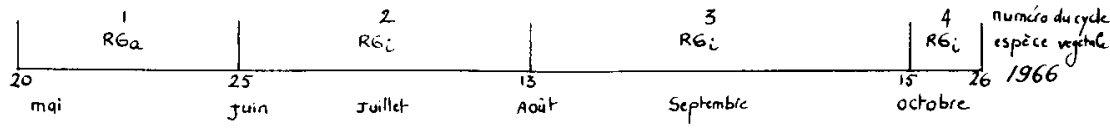

FIG. I. - Calendrier d'exploitation des prairies au cours des différentes années

$$
\begin{aligned}
\mathrm{RG}_{i} & =\text { Ray-grass italien } \\
\mathrm{RG}_{a} & =\text { Ray-grass anglais } \\
\overline{\mathrm{F}} & =\text { Fétuque des prés }
\end{aligned}
$$

\begin{tabular}{|c|c|c|c|c|c|}
\hline Année & $\begin{array}{c}\text { Mode } \\
\text { d'alimentation }\end{array}$ & $\begin{array}{l}\text { Nombre de } \\
\text { bœufs }\end{array}$ & Race & $\begin{array}{l}\text { Poids initial } \\
\qquad(\mathrm{kg})\end{array}$ & Périodes d'étude \\
\hline 1963 & $\begin{array}{c}\text { Pâturage } \\
\text { Herbe fauchée }\end{array}$ & $\begin{array}{l}12 \\
11\end{array}$ & $\begin{array}{l}\text { Charolaise } \\
\text { Charolaise }\end{array}$ & $\begin{array}{l}411 \\
\$ 11\end{array}$ & 6 mai-1/4 septembre \\
\hline $196^{\prime}$ & $\begin{array}{l}\text { Pâturage } \\
\text { Herbe fauchée }\end{array}$ & $18\left\{\begin{array}{l}9 \\
9 \\
9 \\
9\end{array}\right.$ & $\begin{array}{l}\text { Charolaise } \\
\text { Normande } \\
\text { Charolaise } \\
\text { Normande }\end{array}$ & \begin{tabular}{l|l}
406 & 419 \\
430 & 4 \\
403 & 419 \\
433 & 419
\end{tabular} & I mai-2 octobre \\
\hline 1965 & $\begin{array}{c}\text { Pâturage } \\
\text { Herbe fauchée } \\
\text { Herbe fauchée }+ \\
3 \mathrm{~kg} \text { d'orge }\end{array}$ & $\begin{array}{l}15 \\
15 \\
15\end{array}$ & $\begin{array}{l}\text { Normande } \\
\text { Normande } \\
\text { Normande }\end{array}$ & $\begin{array}{l}443 \\
434 \\
447\end{array}$ & 30 avril-1 er octobre \\
\hline 1966 & \begin{tabular}{|} 
Pâturage \\
Herbe fauchée \\
Herbe fauchée + \\
$3 \mathrm{~kg}$ d'orge
\end{tabular} & $\begin{array}{l}15\left\{\begin{array}{r}5 \\
10 \\
5 \\
10 \\
5 \\
15\end{array}\right.\end{array}$ & $\begin{array}{l}\text { Charolaise } \\
\text { Normande } \\
\text { Charolaise } \\
\text { Normande } \\
\text { Charolaise } \\
\text { Normande }\end{array}$ & $\begin{array}{l}\left.\begin{array}{l}425 \\
429\end{array}\right\} 428 \\
449 \\
450 \\
\left.\begin{array}{l}425 \\
439\end{array}\right\} 434\end{array}$ & 21 mai-26 octobre \\
\hline
\end{tabular}

\section{TABLEAU I}

Nombre, race et poids initial des animaux utilisés dans les différents essais 
prairies ont été exploitées après l'année du semis pendant 2 à 4 ans suivant les espèces fourragères. Elles ont reçu 5 tonnes de fumier avant le labour et chaque hiver une fumure phospho-potassique ( $100 \mathrm{~kg}$ de $\mathrm{P}$ et $\mathrm{K}$ ). La fumure azotée a été fractionnée : $50 \mathrm{~kg}$ d'azote au printemps avant le départ de la végétation, et de 40 à $50 \mathrm{~kg}$ après chaque exploitation. Celles-ci ont eu lieu à intervalle de 4 à 8 semaines suivant les espèces, les saisons et les conditions climatiques.

Chaque prairie a été divisée en deux, une partie étant réservée au pâturage, l'autre à la fauche. Il est en effet indispensable d'apporter aux animaux en stabulation une herbe fauchée dans une parcelle qui n'a pas été pâturée lors des exploitations précédentes.

Les bœufs utilisés ont été achetés à l'extérieur, de un à trois mois avant la mise à l'herbe. De race Charolaise ou Normande, ces animaux âgés de 2 à 3 ans étaient maigres et pesaient environ $45^{\circ} \mathrm{kg}$. Dès leur arrivéc au domaine du Pin ces animaux ont été maintenus en stabulation libre couverte ou dans des parcs extérieurs. Ils y ont reçu jusqu'à la mise à l'herke soit du foin (en général de mauvaise qualité) soit de l'ensilage direct, ce qui a permis de les maintenir à peu près à poids constant. Le tableau I donne la répartition par race et le poids initial des animaux suivant les années et les essais.

\section{Conduite des animaux et des prairies}

La mise à l'herbe a été brutale et les animaux sont passés sans transition du régime d'hiver à base de foin ou d'ensilage au régime d'herbe. Les boufs au pâturage ont consommé l'herbe des prairies en pâturage rationné. Les clôtures électriques avant et arrière ont été avancées tous les 4 jours excepté à la fin de l'exploitation des premiers cycles de Ray-grass d'Italie où elles ont été avancées tous les 2 jours lorsque l'herbe est devenue trop haute. La surface allouée à chaque fois a toujours été ajustée pour que les refus correspondent à une sévérité de pâturage normale telle qu'elle avait pu être définie par les expériences de pâturage entreprises au domaine du Pin depuis I958. Les refus laissés par les animaux au pâturage ont été fauchés et enlevés après la Ire et la $2^{\mathbf{e}}$ exploitation. En revanche, les animaux n'ont jamais reçu d'alimentation complémentaire mais avaient à leur disposition des pierres de sel.

Les animaux en stabulation ont toujours reçu l'herbe de la même prairie, exploitée au même stade végétatif et au même âge que les animaux au pâturage. Par groupe de 5 ou 6 , ils ont reçu cette herbe verte à volonté (Io à $\mathrm{r} 5 \mathrm{p}$. Ioo de refus) en 2 repas par jour à 8 heures et $\mathrm{r}_{4}$ heures et les refus ont été enlevés une fois par jour avant la distribution du premier repas de la journée. Dans une partie réservée à la fauche, l'herbe nécessaire aux animaux a été fauchée chaque matin, sauf le dimanche, avec une faucheuse classique portée par un tracteur et ramassée par une remorque auto-chargeuse. Pour une prairie donnée, le temps d'exploitation a été fixé par le mode d'exploitation qui progressait le plus vite, l'excédent d'herbe dans la partie pâturéc ou dans la partie réservée à la fauche a été fauché, estimé et transformé soit en foin soit cn ensilage.

Nous avons été obligés à plusieurs reprises d'utiliser en cours d'expérience des prairies non prévues au départ parce que la repousse de l'herbe sur les prairies prévues était insuffisante, ou que celles-ci étaient trop humides à certains moments. C'est ce qui explique que la nature de l'herbe utilisée a varié souvent même à l'intérieur d'un même cycle de végétation (fig. I).

Les animaux ont été maintenus en expérience jusqu'à ce qu'ils aient atteint un état d'engraissement optimum. En fait il a fallu étaler dans une certaine mesure la période d'abattage des animaux ayant atteint cet état pour assurer un fonctionnement régulier de l'abattoir du C. N. R. Z.

\section{Mesures}

Les mesures ont commencé chaque année dans la première décade de mai alors que la végétation avait démarré bien avant car sous le climat très humide du Pin il n'est pas possible de faucher mécaniquement de l'herbe avant cette date, le sol ne "portant " pas, et les machines de récolte (tracteurs et remorques) creusant de profondes ornières qui abîment la prairie.

\section{Gains de poids des animaux.}

Au début de l'exploitation des prairies les animaux en stabulation ont été pesés tous les jours et les animaux au pâturage tous les 2 jours jusqu'au moment où le poids des animaux a cessé de diminuer. Le poids moyen journalier le plus faible enregistré a été choisi comme poids initial du lot, les poids individuels des animaux lors de cette pesée comme poids initial de chaque animal. Les animaux ont ensuite été pesés à la fin de chaque cycle de végétation et au moment de leur départ pour l'abattoir. Ces pesées ont permis de calculer les gains de poids vif par cycle et pour la durée de l'expérience. Les animaux étant partis progressivement à l'abattoir, il devient bien souvent illusoire de comparer au cours des 4 e cycles, les croîts individuels et les gains de poids vif à l'hectare, car le nombre d'animaux restant en expérience devient trop faible et diffère d'un traitement à l'autre. 
Une difficulté résulte du fait que les poids des animaux au pâturage et en stabulation ne sont pas comparables. En effet après chaque cycle de végétation le poids des animaux au pâturage a été estimé commeé tant la moyenne de deux peséeś, l'une effectuéc le matin vers 9 heures après un cycle de pâturage, l'autre l'après-midi vers I6 heures, les animaux étant privés de nourriture et d'eau depuis la pesée du matin. En revanche, le poids des animaux en stabulation est la moyenne des peséss effectuées pendant 2 ou 3 jours consécutifs à I 3 heures, les animaux disposant toujours d'hr'sz et d'exu. Seuls les gains de poids sont donc comparables avec cette restriction cependant que pour les animaux au pâturage le poids de début d'expérience est la moyenne des pesées de 2 ou 3 jours, les animaux ayant été pesés une seule fois par jour à 9 heures après un cycle de pâturaga. Le gain de poids vif réalisé par les animaux au pâturage au cours du premier cycle de végétation a donc été sous-estimé. I.'étude des variations du contenu digestif des bœufs au pâturage réaliséc par BÉRANGER (résultats non publiés) permet de chiffrer à 5 ou $6 \mathrm{~kg}$ cette sous-estimation.

\section{Production végétale.}

La quantité de matière sèche produite à chaque cycle par les prairies pâturées a été estimée grossièrement par pesée chaque scmaine, juste avant l'entrée des animaux sur la parcelle, d'un échantillon fauché sur une surface de $25 \times 1 \mathrm{~m}$; et celle produite par les prairies fauchées par pesée sur un pont-bascule des quantités d'herbe récoltée chaque jour.

\section{Quantité d'herbe ingérée et digestibilité d' l'herbe fauchée.}

Los quantités de matière verte et de matière sèche ingérées par chaque lot en stabulation ont été mesurés chaque jour, sauf le dimanche, par pesée des quantités distribuées et refusées et détermination de la teneur en matière sèche de l'herbe de chaque repas et des refus.
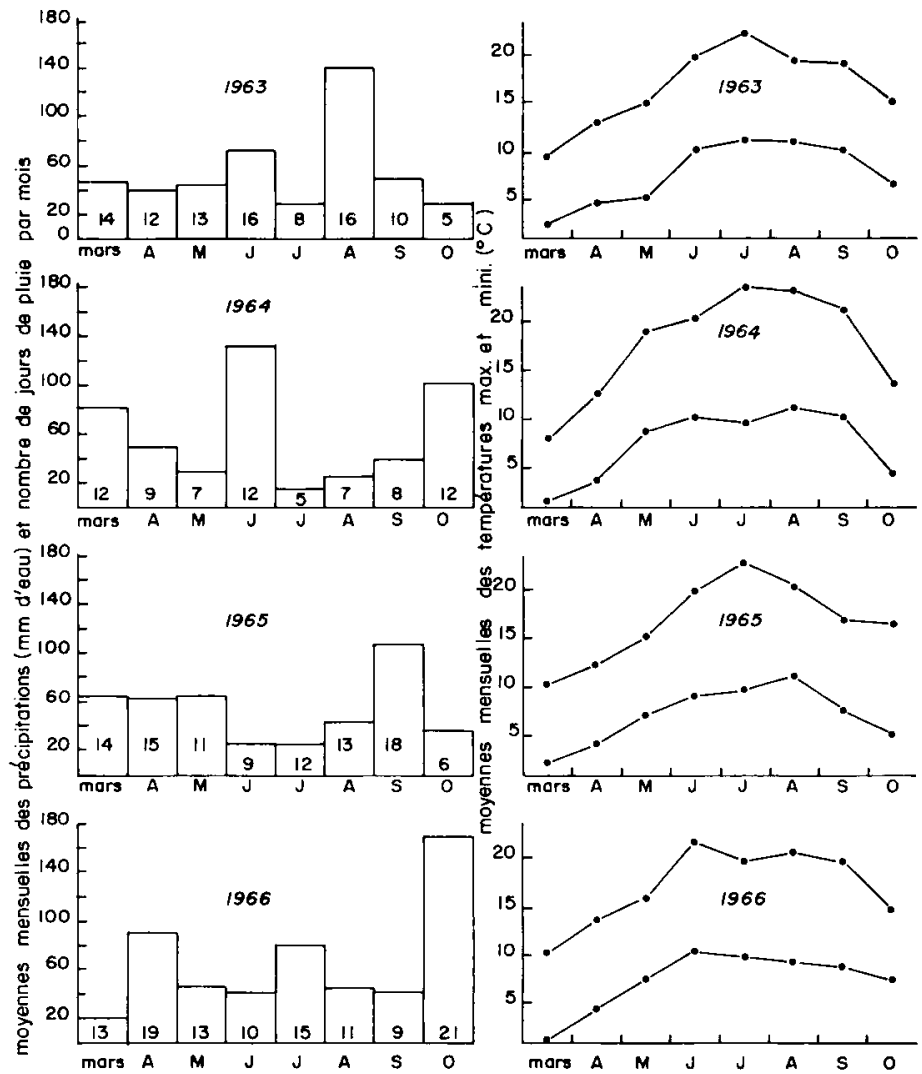

FIG. 2. - Moyennes mensuelles des précipitations (hauteur d'eau et nombre de jours de pluie) et des températures maxima et minima au cours des 4 années d'expérience 
A partir de 1964, la digestibilité de l'herbe distribuée aux boufs en stabulation a été mesurée sur un lot de 4 béliers. La méthode de mesure employée a déjà été exposée dans une publication précédente (Demarquilly et Jarrige, I964).

On a dosé les teneurs en cendres, en azote et en celliulose brute (cellullose Weende) des échantillons représentatifs de l'herbe coupée constitués lors de chaque période de digestibilité sur moutons. La valeur énergétique nette de l'herbe distribuée aux boeufs en stabulation a été estimée par la formule de BREıREM (1954) à partir des teneurs en matière organique digestible (MOD en $g$ par $\mathrm{kg}$ de matière sèche) et en matière organique non digestible (MOND en $\mathrm{g}$ par kg de matière sèche) mesurées sur les moutons.

$$
\mathrm{UF} / \mathrm{kg} \mathrm{MS}=\frac{2,36 \mathrm{MOD}-\mathbf{1 , 2 0} \mathrm{MOND}}{\mathrm{I} 65^{\circ}}
$$

Nous avons relevé les données climatiques; elles ont été très différentes suivant les années (fig. 2).

\section{RÉSULTATS}

\section{Composition chimique et digestibilité}

Nous avons mesuré la composition chimique et la digestibilité de tous les fourrages utilisés en I964, I965 et r966 soit 27 échantillons de Ray-grass italien, 6 échantillons de Ray-grass anglais (tous du I er cycle) et 27 échantillons de Fétuque des prés. Les résultats sont présentés dans la figure 3 dans laquelle les échantillons provenant de la même exploitation d'une même prairie ont été joints en traits pleins.

Compte tenu du fait que les mesures ont commencé relativement tard au printemps, la teneur en matière sèche des échantillons des fourrages du premier cycle n'a jamais été inférieure à $I_{5,5} \mathrm{p}$. Ioo et a augmenté relativement peu avec l'âge (5 points au maximum). Elle a, au contraire, été variable pour les repousses (I2,9 à $3^{6, \text { I }}$ p. roo) reflétant les variations climatiques de l'année ou de la saison.

Au cours du premier cycle de croissance, la composition de la matière sèche des fourrages utilisés a présenté l'évolution caractéristique associée aux modifications morphologiques des graminées. La teneur en matières azotées est passée de I5 à 8 p. Ioo environ, la teneur en cellulose brute a augmenté de 22 à 33 p. Ioo environ. Le coefficient de digestibilité de la matière organique a diminué de 80 à $65 \mathrm{p}$. Ioo entre le début mai et le I 5 juin. Il a évolué de façon relativement semblable pour les différentes prairies, c'est-à-dire qu'il a diminué de façon assez rapide et régulière (en moyenne 3 points par semaine). A une date donnée il présente des variations importantes suivant les années, alors qu'il n'y a pas de différence de composition chimique. Ces différences entre années sont dues aux espèces végétales utilisées et à leurs précocités différentes ; c'est ainsi que le Ray-grass anglais a été systématiquement le plus digestible mais il s'agissait de la variété Melle-pâture qui est beaucoup plus tardive que les Fétuques des prés ( $S 215$ et Naïade) et surtout que le Ray-grass d'Italie (Rina) utilisés. En revanche, toujours à une date donnée, les différences entre années ont été très faibles pour une même espèce fourragère.

La teneur en matières azotées des repousses a été plus variable que celle des fourrages du I er cycle $(8,7$ à I9,5 p. Ioo) et a eu tendance à augmenter au cours de l'année, notamment à partir du $4^{\mathrm{e}}$ cycle, ce qui est normal, les fourrages étant de plus en plus feuillus (fig. 3). En revanche, la teneur en cellulose brute des repousses a moins varié qu'au cours du I ${ }^{\text {er }}$ cycle et a presque toujours été comprise entre 24 et 30 p. roo avec une tendance à diminuer au cours des cycles successifs. Elle a été sys- 

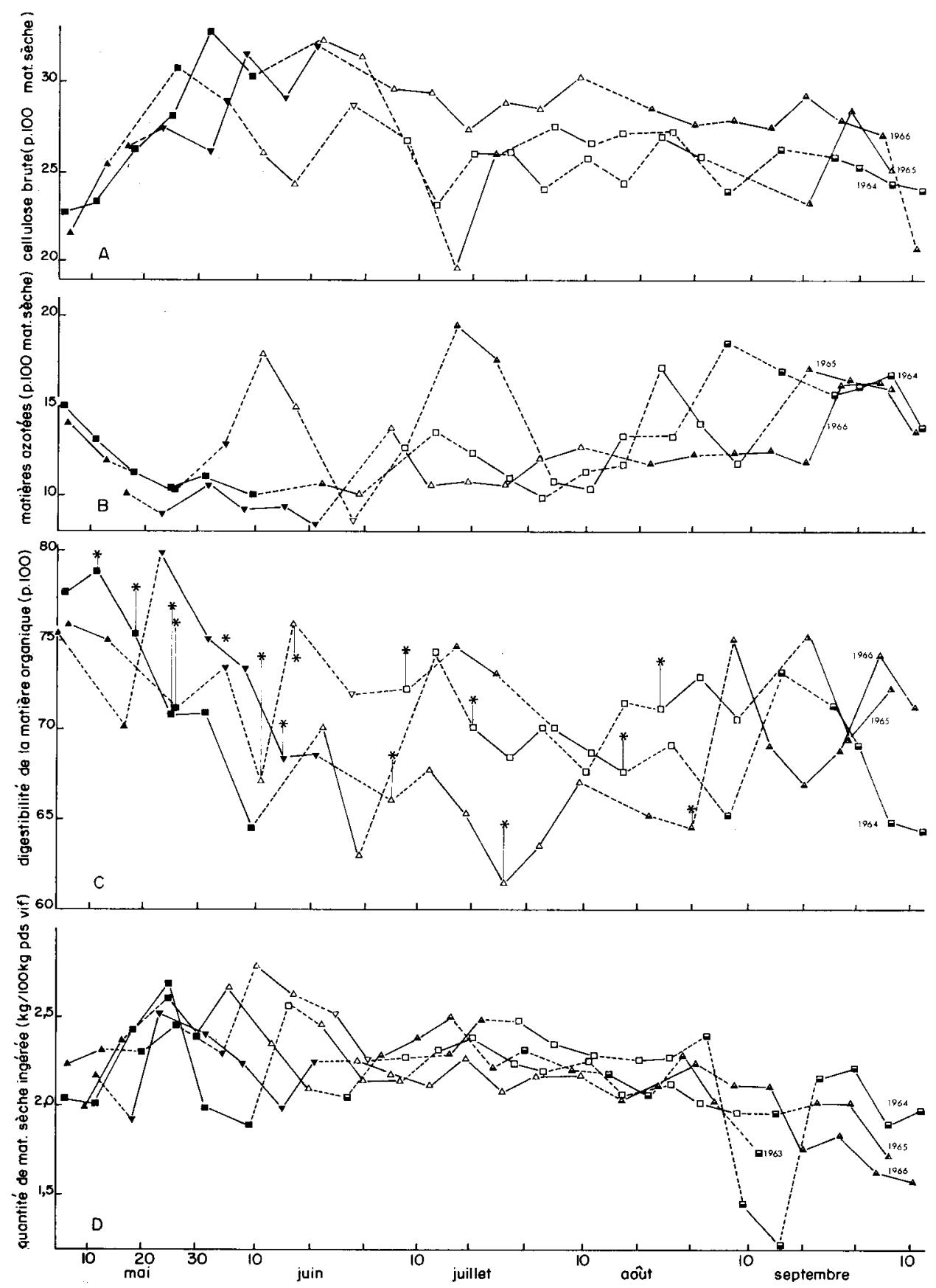

FIG. 3. - Teneur en cellulose brute (A), teneur en matières azotées (B) Coefficient de digestibilité de la matière organique (C), et quantité de matière sèche ingérée (D) des fourrages utilisés en 1964, 1965 et 1960

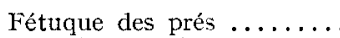

Ray-grass anglais $\ldots \ldots \ldots \ldots$

Ray-grass italien ..........

$\begin{array}{cccc}\mathrm{I}^{\mathrm{er}} \text { cycle } & 2^{\mathrm{e}} \text { cycle } & 3^{\mathrm{e}} \text { cycle } & 4^{\mathrm{e}} \text { cycle } \\ \mathbf{\nabla} & \square & \mathbf{a} & \mathbf{a} \\ \mathbf{\nabla} & \nabla & \nabla & \mathbf{\nabla} \\ \mathbf{\square} & \triangle & \mathbf{A} & \mathbf{\Delta}\end{array}$


tématiquement plus élevée en I966 où la majorité des repousses utilisées étaient des Ray-grass d'Italie qui, par opposition à la Fétuque des prés, contiennent toujours beaucoup de tiges. La digestibilité de la matière organique des repousses a varié dans des limites assez larges (6I,4 à 76,0 p. IOo) mais sans obéir à des lois systématiques.

On peut dire cependant que d'une façon générale la digestibilité de l'herbe fauchée a été bonne (environ $70 \mathrm{p}$. Ioo) et a relativement peu varié ; elle a été comprise entre 65 et $75 \mathrm{p}$. Ioo si on excepte le début des premiers cycles et quelques repousses épiées du $2^{\mathrm{e}}$ cycle. Elle a, en tout cas, beaucoup moins varié que lors des études effectuées sur les prairies du C. N. R. Z. à Jouy-en-Josas et à la Minière (DEMarquilix et JARRIGE, I964). Cela est dû au faible nombre (3) des espèces utilisées au Pin, celles-ci étant en outre de digestibilité assez comparable au même stade végétatif ou au même âge.

Nous avons comparé la teneur en azote des fèces des bœufs en stabulation ne recevant que de l'herbe et des bœufs au pâturage pour essayer d'avoir une idée du tri effectué au pâturage. Ces comparaisons ont été faites 2 fois aux ${ }_{\mathrm{I}}^{\mathrm{er}}, 2^{\mathrm{e}}$ et $3^{\mathrm{e}}$ cycles en Ig64 et I965. Les échantillons représentatifs des fèces des bœufs au pâturage ont été obtenus en échantillonnant chaque jour durant une semaine toutes les bouses fraîches sur la prairie ; ceux des bœufs en stabulation ont été obtenus par mélange des échantillons de fèces prélevés directement dans le rectum deux fois par jour pendant une semaine. Ia teneur en azote des fèces des bœufs au pâturage a été plus élevée que celle des bœufs en stabulation. La digestibilité de l'herbe pâturée a donc été plus importante que celle de l'herbe fauchée parce que les bœufs au pâturage ont effectué un tri. L,es valeurs de la digestibilité de l'herbe pâturée, estimées à partir des teneurs en azote des fèces, ont été figurées par des astérisques sur la figure 3. Si on compare ces valeurs estimées aux valeurs correspondantes mesurées sur les moutons on constate que le tri effectué au pâturage a été d'autant plus important que la qualité de l'herbe diminuait, notamment au cours du premier cycle de végétation.

\section{Crô̂ts individuels et durée d'engraissement}

Nous avons calculé les croîts moyens de deux façons :

- pour la période pendant laquelle tous les animaux sont en expérience; ces croits ne correspondent pas à la durée totale de l'expérience mais ils permettent de comparer les lots de façon rigoureuse ;

- par jour moyen de présence ; ces croîts correspondent donc à la durée totale de l'expérience y compris la période pendant laquelle certains animaux déjà gras étaient partis pour l'abattoir, les autres étant maintenus en expérience soit parce qu'il nous a fallu étaler la période d'abattage soit parce que quelques animaux n'avaient pas encore atteint un état d'engraissement suffisant.

Ces croîts sont regroupés dans le tableau 2 avec les durées d'engraissement et les résultats d'abattage.

I L Les croîts réalisés par jour de présence ont été compris suivant les années entre 884 et I I $52 \mathrm{~g}$ par jour au pâturage et entre 9 I 5 et I I $43 \mathrm{~g}$ par jour en stabulation. Les différences entre le pâturage et la stabulation ne sont pas significatives à l'exception, toutefois, de l'année I964 parce que le lot en stabulation avait reçu au 
$4^{\mathrm{e}}$ cycle une herbe de mauvaise qualité (herbe pâturée précédemment). Les croîts moyens individuels par jour de présence pour l'ensemble des quatre essais sont d'ailleurs très voisins pour les animaux au pâturage ou en stabulation ( $1067 \mathrm{~g}$ et I $039 \mathrm{~g}$ respectivement).

Lorsqu'on considère les croîts réalisés pendant la période où tous les animaux sont présents, les différences ne sont pas non plus significatives, quelle que soit l'année de l'essai.

Les variations importantes des croîts observées d'une année à 1'autre sont dues essentiellement à l'année I966 où les croîts ont été plus faibles en raison d'un premier cycle difficile (parcelles gorgées d'eau, d'herbe souillée et mal consommée).

D'une façon générale les croîts réalisés au cours du premier cycle de végétation ont été très élevés mais aussi très variables suivant les années : I $594 \mathrm{~g} /$ jour en moyenne ( $\mathrm{I}$ 098 à $2098 \mathrm{~g}$ suivant les années) en stabulation, I $305 \mathrm{~g} /$ jour en moyenne ( 852 à I 62 I g suivant les années) au pâturage. Ils sont significativement plus élevés en stabulation qu'au pâturage sauf en I 964 .

La digestibilité de l'herbe pâturée ayant été un peu supérieure à celle de l'herbe fauchée, la croissance inférieure au pâturage ne peut résulter que d'une consommation plus faible. Cela est certes possible car compte tenu de la pluviométrie du Pin et de la nature du sol, il a fallu exploiter des fourrages assez hauts au printemps, donc allouer tous les 2 ou 4 jours une surface restreinte aux animaux, surface dont l'herbe était assez vite couchée et souillée par les pieds des animaux pendant les périodes pluvieuses. Les bœufs au pâturage n'ont donc pas toujours eu une quantité suffisante d'herbe saine à leur disposition et se sont peut-être habitués moins rapidement à l'herbe, la mise à l'herbe correspondant en même temps pour eux à un changement de mode de vie. Il est cependant possible que ces différences soient artificielles et dues simplement à une mauvaise estimation des croissances réelles. Nous avons vu, en effet, que les gains de poids des animaux au pâturage au cours du $\mathrm{I}^{\mathrm{er}}$ cycle avaient été vraisemblablement sous-estimés d'environ 5 à $6 \mathrm{~kg}$. S'il en est vraiment ainsi, les croissances journalières réelles devraient être supérieures de $200 \mathrm{~g}$ environ à celles calculées.

En revanche, les croîts réalisés au cours des deuxièmes cycles ont été plus élevés au pâturage qu'en stabulation; en moyenne I I $8 \mathrm{~g} / \mathrm{jour}$ contre $973 \mathrm{~g} / \mathrm{jour}$, les différences étant significatives en I964, I965 et I966. Il en est de même de ceux réalisés pendant les troisièmes cycles; en moyenne I $108 \mathrm{~g} / \mathrm{jour}$ contre $\mathrm{I}$ o3o g/jour mais les différences ne sont significatives qu'en 1964 .

Les durées d'engraissement ont varié suivant les années de I3I à I 55 jours en stabulation et de I28 à I55 jours au pâturage, les différences entre les traitements n'étant pas significatives.

Les durées moyennes d'engraissement calculées pour l'ensemble des quatre essais ne sont pas non plus significativement différentes ( 147 et 144 jours).

Durant les 4 années, I 2 animaux (soit $20 \mathrm{p}$. Ioo des animaux) n'ont pas été " finis » en stabulation et 7 animaux (soit $x 2$ p. Ioo) au pâturage.

$2^{\circ}$ L'apport de $3 \mathrm{~kg}$ d'orge aux animaux en stabulation s'est traduit par une augmentation du croît moyen qui n'est significative $(P \leqslant 0,05)$ qu'en Ig66 et seulement lorsqu'on considère les croîts calculés sur la durée moyenne de l'expérience.

Les animaux recevant le complément d'orge ont réalisé des croîts très élevés au $\mathrm{I}^{\text {er }}$ cycle (I $736 \mathrm{~g} / \mathrm{jour}$ en I965, I 4I9 g/jour en I966) et significativement supérieurs

Annales de Zootechnie. - 1969 . 
à ceux réalisés par les animaux en stabulation en r966. Lors des deuxièmes cycles les croîts des animaux recevant l'orge ont été significativement plus élevés que ceux des animaux recevant uniquement de 1 'herbe en stabulation mais plus faibles que ceux des animaux au pâturage, les différences n'étant cependant pas significatives. Lors du troisième cycle de I 966 , leurs croîts ont été légèrement plus faibles que ceux des autres animaux.

L'apport de $3 \mathrm{~kg}$ d'orge s'est surtout traduit par une diminution significative $(P \leqslant 0,0 I)$ de la durée d'engraissement (qui a été de II 7 jours en I 965 , et de 122 jours en I966) par rapport à celle des animaux au pâturage (I28 et I 50 jours) et en stabulation (I3I et I44 jours).

\section{État d'engraissement}

L'état d'engraissement a été apprécié à l'abattage par la proportion de gras périrénal et de gras périrénal et précrural dans la carcasse. Nous avons ajouté à ces critères des notes d'appréciation subjective de l'état d'engraissement des animaux et le rendement vrai (1) (tab1. 2). Les carcasses des animaux au pâturage et des animaux en stabulation n'ont pas présenté de différences significatives, il en a été de même des carcasses des animaux ayant reçu le complément de $3 \mathrm{~kg}$ d'orge par rapport à celles des deux autres lots. Cela est normal puisqu'on s'est efforcé d'abattre les animaux à un état d'engraissement identique.

\section{Productions à l'hectare}

En moyenne les prairies fauchées ont produit 8,2 et 8,8 tonnes de matière sèche respectivement en I964 et I965 (tab1. 3). Remarquons que la production du premier cycle a représenté en moyenne $45 \mathrm{p}$. Ioo de la production cumulée des $\mathrm{I}^{\mathrm{er}}, 2^{\mathrm{e}}$ et $3^{\mathbf{e}}$ cycles (tab1. 3 ).

La quantité de matière sèche produite par les prairies pâturées n'a pas pu être estimée de façon aussi correcte que celle des prairies fauchées. La valeur estimée n'a cependant pas été significativement différente de la quantité de matière sèche produite par les prairies fauchées (tabl. 3).

Compte tenu de la bonne productivité des prairies utilisées, le chargement à l'hectare a été élevé ; il a été en moyenne pour la période où les animaux sont encore tous présents de 5,0 animaux/ha ( 3,6 à 6,5 suivant les années) pour les prairies exploitées par la fauche et de 4,8 animaux/ha (3,9 à 5,9 suivant les années) pour les prairies pâturées. Il est donc relativement peu différent entre les 2 traitements. Les différences entre les traitements sont plus importantes mais ne peuvent plus être comparées si on considère le chargement moyen pendant la totalité de l'expérience : 5 , I (4, I à 6,4 suivant les années) en affouragement et $4,5(3,7$ à 5,4$)$ au pâturage.

L'apport de $3 \mathrm{~kg}$ d'orge par jour a permis d'augmenter sensiblement ( $\mathrm{I}, 5$ animaux/ha) le nombre d'animaux alimentés en stabulation.

S'il a été possible d'alimenter en moyenne par saison de pâturage 3,7 à 6,4 animaux à l'hectare au pâturage et en stabulation, le chargement a en fait varié (de 2 à II animaux à 1'hectare) suivant le cycle végétatif et l'année. Le chargement le

(1) $\frac{\text { Poids de carcasse }}{\text { Poids vif vide }} \times$ Ioo 


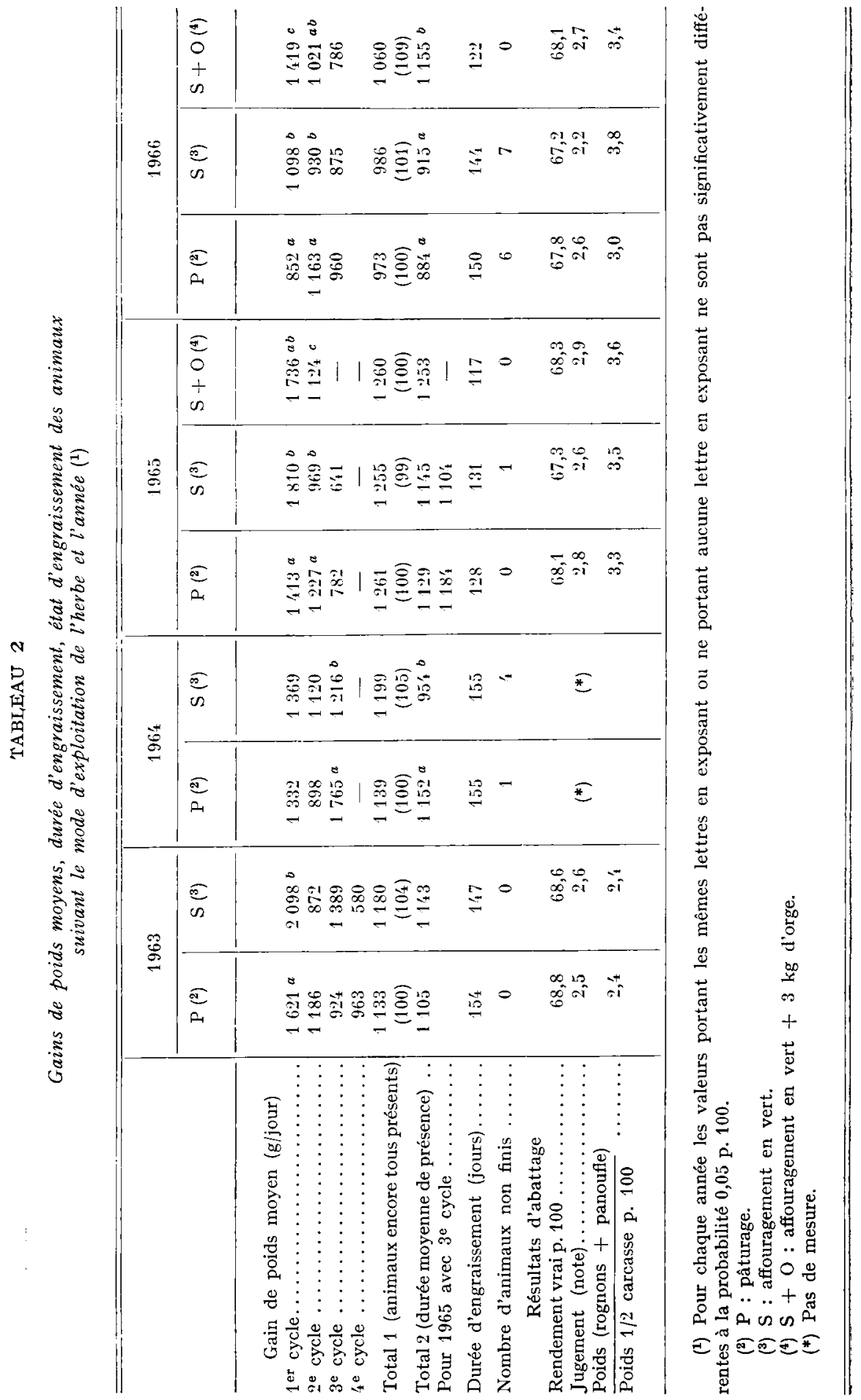


M. CHENOST, C. DEMARQUII,YY

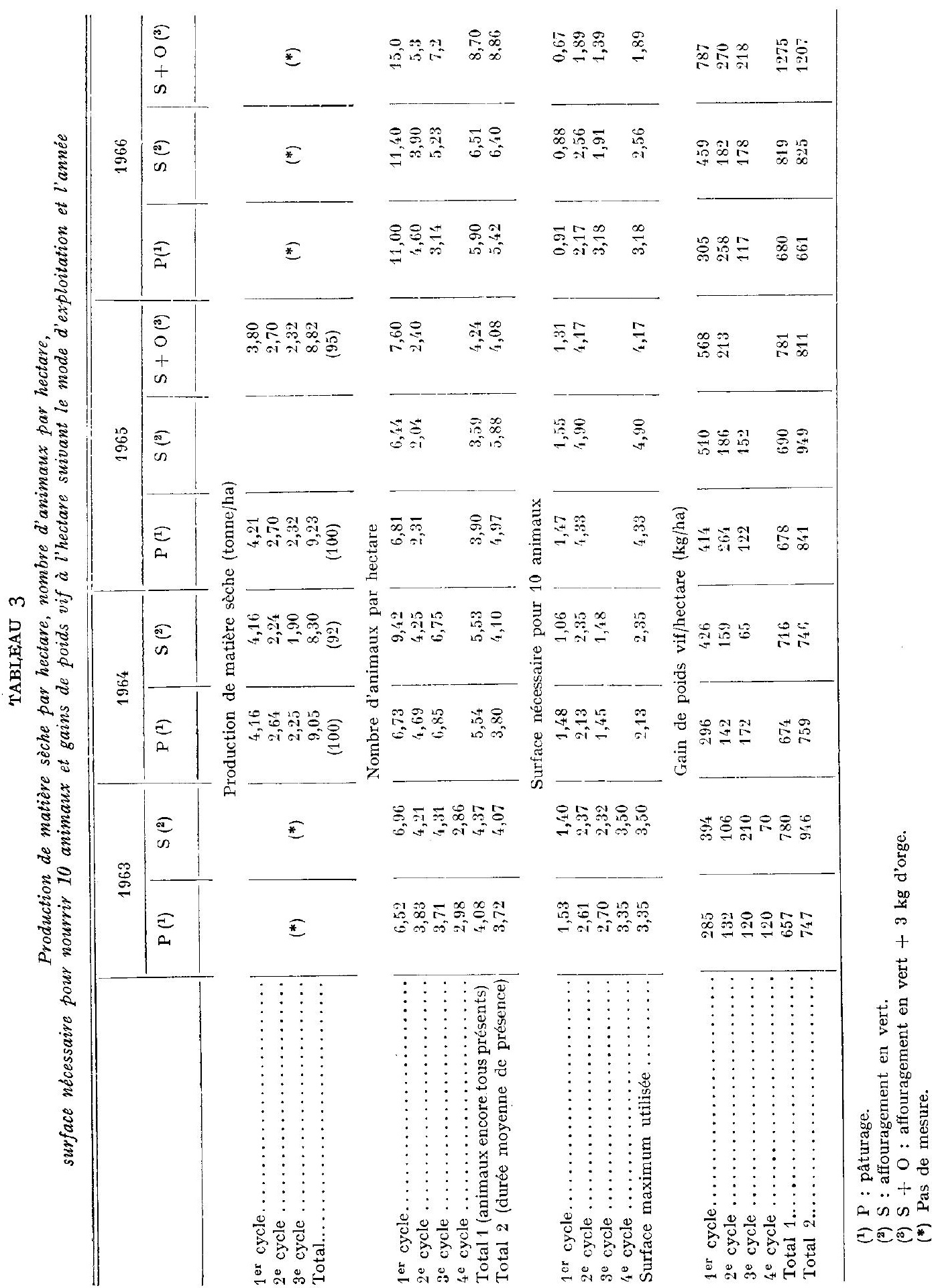


plus élevé correspond chaque année au premier cycle de végétation et il diminue ensuite au fur et à mesure des cycles successifs. Il peut être utile de faire ressortir de nos résultats la surface nécessaire à prévoir pour alimenter sans jamais manquer d'herbe ro animaux pendant une saison de pâturage ; elle est en moyenne de 3,25 ha $(2$, I 5 à 4,35$)$ au pâturage et de 3,35 ha $(2,35$ à 4,90$)$ en affouragement en vert seul et de 3,05 (I,90 à 4,I 5) quand les animaux reçoivent une complémentation de $3 \mathrm{~kg}$ d'orge.

De la mise à l'herbe jujqu'au début septembre ( $\mathrm{I}^{\mathrm{er}}$ au 20 septembre suivant les années), date du départ des premiers animaux pour l'abattoir, le gain de poids vif obtenu à l'hectare a été élevé. Il a été en moyenne de $672 \mathrm{~kg}(657$ à 680) par hectare pâturé et de $75 \mathrm{I} \mathrm{kg}(690$ à $8 \mathrm{Ig})$ par hectare fauché. L'affouragement en vert a donc permis de produire $79 \mathrm{~kg}$ (I 2 à I39) de gain de poids vif à l'hectare soit II,8 p. IOO de plus que le pâturage, la valeur la plus faible ayant été enregistrée en I965 où le. fourrage utilisé a été presque exclusivement de la Fétuque des prés. L'apport de $3 \mathrm{~kg}$ d'orge a permis d'augmenter le gain de poids vif par hectare fauché de $\mathrm{I} 7,0$ et 55,0 p. roo respectivement en I965 et I966 (tabl. 3). Quant au gain de poids vif par saison de pâturage, il est encore plus élevé ; $752 \mathrm{~kg}$ par hectare pâturé et $866 \mathrm{~kg}$ par hectare fauché.

Les gains de poids vif réalijés au premier cycle représentent suivant les années de 43,3 à $6 \mathrm{I}, 0 \mathrm{p}$. I oo du gain total réalisé en trois exploitations au pâturage et de 50,5 à 73,9 p. Ioo du gain total réalisé en stabulation.

Les gains de poids vif réalisés au premier cycle ont toujours été beaucoup plus élevés en stabulation qu'au pâturage ( 447 contre $325 \mathrm{~kg}$ ) alors que ceux réalisés au $2^{\mathrm{e}}$ cycle ont été plus faibles ( 58 contre $\mathrm{I} 99 \mathrm{~kg}$ ). Ce fait peut s'expliquer facilement : au I ${ }^{\text {er }}$ cycle l'herbe est très digestible, le tri effectué au pâturage est peu important mais les refus dus aux bouses et au piétinement deviennent vite importants quand 1'herbe devient haute. En revanche, le tri effectué au pâturage est plus efficace au $2^{\mathbf{e}}$ cycle et les animaux au pâturage exploitent mieux qu'une barre de coupe une herbe moins fournie, surtout quand les prairies qu'ils exploitent n'ont pas été pâturées au $\mathrm{I}^{\mathrm{er}}$ cycle, ce qui était le cas pour la majorité des prairies en deuxième exploitation.

L'apport de $3 \mathrm{~kg}$ d'orge a permis d'augmenter les gains de poids vif par hectare fauché de façon très variable suivant les années et le numéro du cycle de végétation ; respectivement en I 965 et Ig66 de II,4 et 7I,4 p. Ioo au premier cycle et de 14,5 et 48,5 p. roo au deuxième cycle.

D'une façon générale on peut dire que les différences de gain de poids vif réalisé à l'hectare suivant les différents traitements (pâturage, affouragement en vert, affouragement + complémentation) et même suivant les années pour un traitement donné, étaient acquises à la fin du premier cycle de végétation.

\section{Quantités ingérées}

Nous avons mesuré les quantités d'herbe ingérées par les bœufs en stabulation lors de chaque exploitation des prairies utilisées de I963 à Ig66. Quand 1'exploitation d'une même prairie a eu une durée égale ou supérieure à 2 semaines, la quantité ingérée a été calculée par période d'une semaine. Dans le cas contraire, la quantité ingérée a été calculée pour la durée de l'exploitation (5 à I3 jours). 


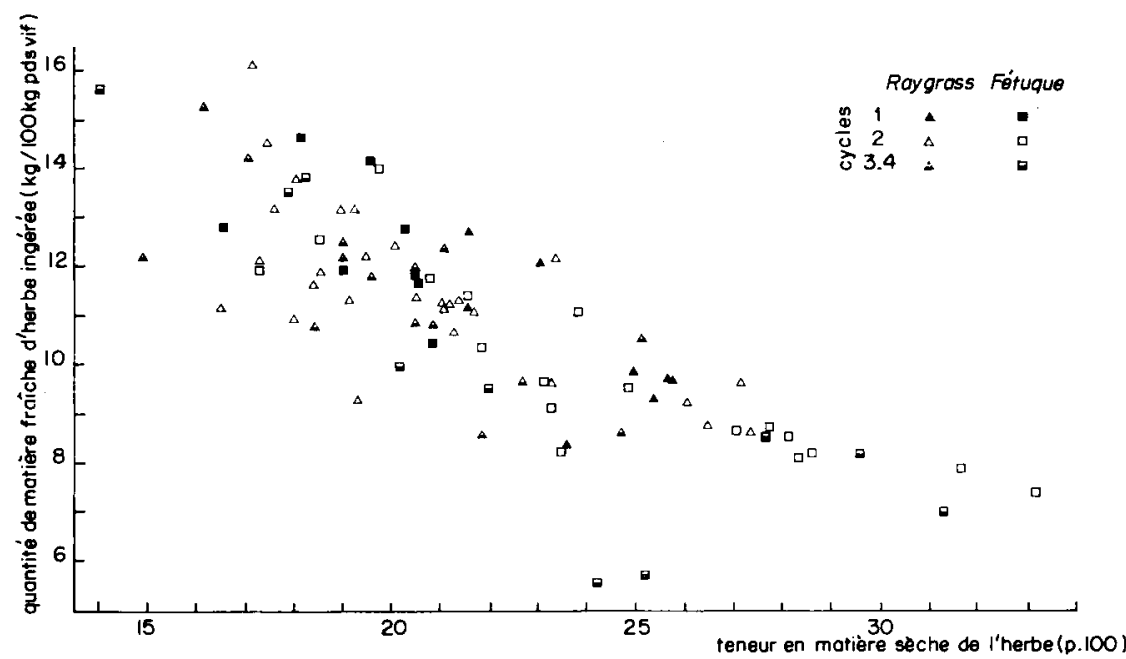

FIG. 4. - Relation entre la quantité de matière frâ̂che d'herbe ingérée et la teneur en matière sèche de l'herbe

\section{TABIEAU 4}

Quantités (en $\mathrm{kg}$ ) moyennes de matière sèche ingérées par jour et par $100 \mathrm{~kg}$ de poids vif suivant le numéro du cycle de végétation et l'espèce fourragère

\section{Pour l'ensemble des 4 années}

\begin{tabular}{|c|c|c|c|c|c|c|}
\hline \multirow[b]{2}{*}{$\begin{array}{l}\text { Numéro } \\
\text { du cycle }\end{array}$} & \multicolumn{2}{|c|}{ Ray-grass } & \multicolumn{2}{|c|}{ Fétuque des prés } & \multicolumn{2}{|c|}{ Total (toutes les espèces) } \\
\hline & $\begin{array}{l}\text { Nombre } \\
\text { d'échan- } \\
\text { tillons }\end{array}$ & $\begin{array}{c}\text { Quantité } \\
\text { ingérée } \\
\text { moyenne }\end{array}$ & $\begin{array}{l}\text { Nombre } \\
\text { d'échan- } \\
\text { tillons }\end{array}$ & $\begin{array}{l}\text { Quantité } \\
\text { ingérée } \\
\text { moyenne }\end{array}$ & $\begin{array}{l}\text { Nombre } \\
\text { d'échan- } \\
\text { tillons }\end{array}$ & $\begin{array}{l}\text { Quantité } \\
\text { ingérée } \\
\text { moyenne }\end{array}$ \\
\hline $1 \ldots$ & 9 & 2,26 & 9 & 2,31 & 18 & 2,28 \\
\hline $2 \ldots$ & 15 & 2,33 & 16 & 2,42 & 31 & 2,30 \\
\hline $3 \ldots$ & 17 & 2,14 & 7 & 1,79 & $\because 1$ & 2,12 \\
\hline $4 \ldots$ & 9 & 2,09 & 3 & 1,99 & 12 & 2,06 \\
\hline Total........ & 50 & 2,21 & 35 & 2,23 & & \\
\hline
\end{tabular}

2. Pour une année donnée

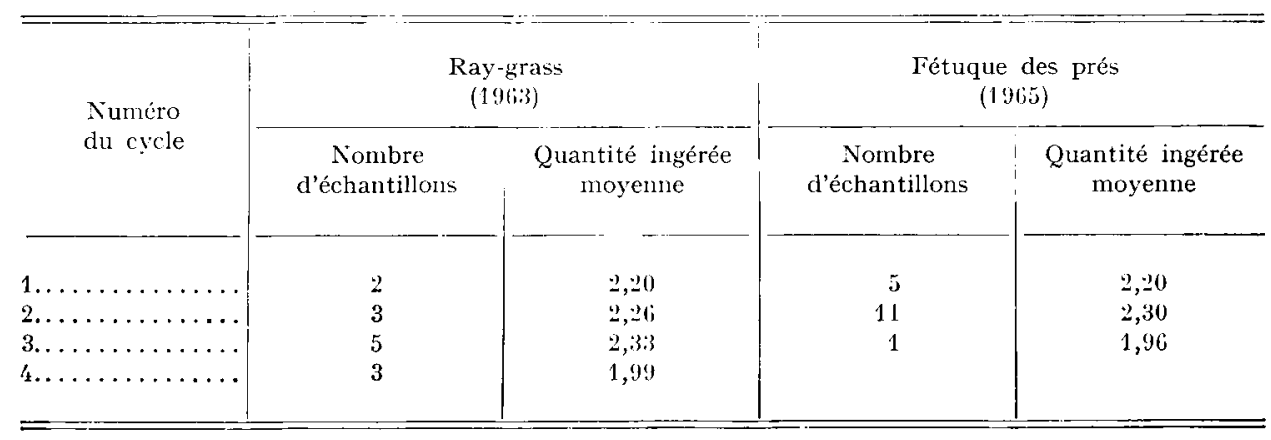


Quantité d'herbe ingérée.

Exception faite d'une période aberrante en 1965 pendant laquelle les animaux en stabulation ont reçu de l'herbe d'une prairie ayant été précédemment pâturée, les quantités de matière fraîche ingérées ont varié de 7,8 à I6,I $\mathrm{kg}$ par Ioo $\mathrm{kg}$ de poids vif et les quantités de matière sèche ingérées de 1,57 à $2,90 \mathrm{~kg}$ par $100 \mathrm{~kg}$ de poids vif suivant les fourrages distribués au cours des 4 années de mesure.

Les quantités de matière fraîche ingérées ont diminué quand la teneur en matière sèche de 1'herbe augmentait (fig. 4).

Les quantités de matière sèche ingérées ont été indépendantes de la digestibilité et de la teneur en matière sèche de l'herbe quand on considère l'ensemble des échantillons. Cependant pour un échantillon donné, la quantité de matière sèche ingérée chaque jour a varié dans le même sens que la teneur en matière sèche de l'herbe fauchée chaque matin. En moyenne égale à 2,2I $\mathrm{kg}$ par Ioo $\mathrm{kg}$ de poids vif, la quantité de matière sèche ingérée a présenté le maximum de variation au début et à la fin des essais et a eu tendance à diminuer avec le numéro de végétation (fig. 3). Cependant, les $2^{\mathrm{e}}$ cycles ont été en moyenne les mieux consommés et cela que l'on considère toutes les espèces utilisées ou une espèce donnée pour 1'ensemble des 4 années ou pour une année donnée (tabl. 4). Les quantités de Ray-grass et de Fétuque des prés

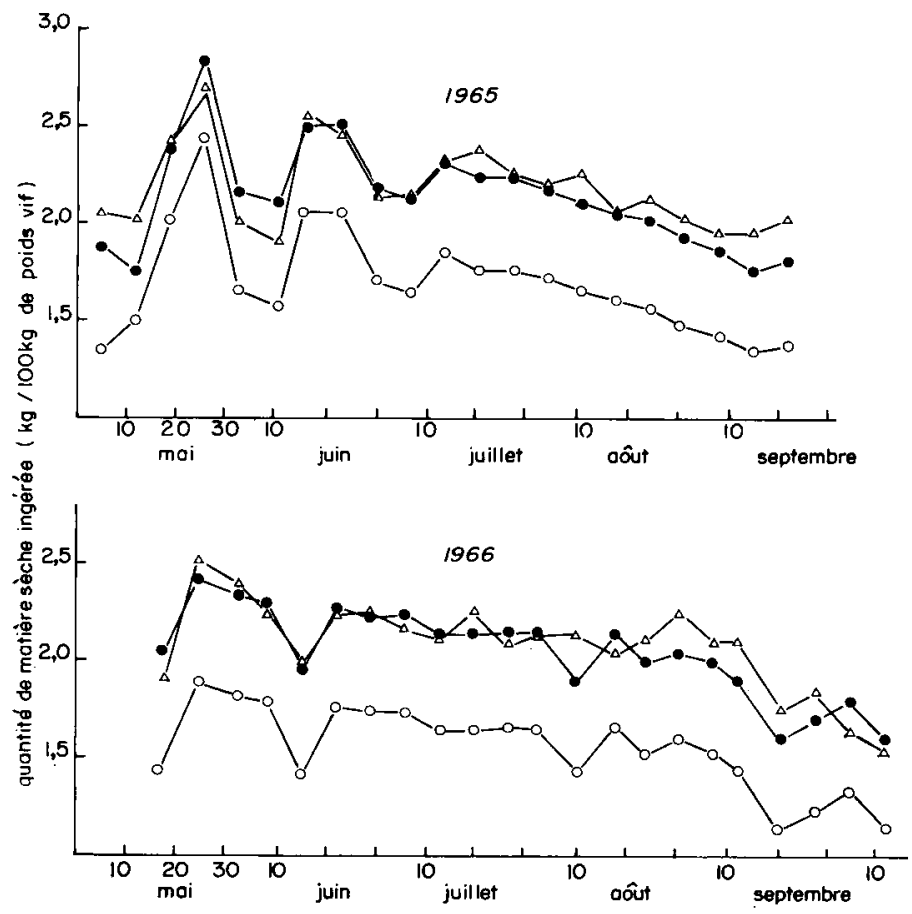

FIG. 5. - Comparaison entre les quantités de matière sèche ingérées par les baufs ne recevant que de l'herbe et celles ingérées par les bceufs recevant de l'herbe et $3 \mathrm{~kg}$ d'orge par jour

$\Delta$ Matière sèche d'herbe ingérée par les boufs ne recevant que de l'herbe

- Matière secche d'herbe ingérée par les boufs recevant de l'herbe et $3 \mathrm{~kg}$ d'orge

- Matière seche totale ingérée par les boufs recevant de l'herbe et $3 \mathrm{~kg}$ d'orge 
M. CHENOST, C. DEMARQUILLY
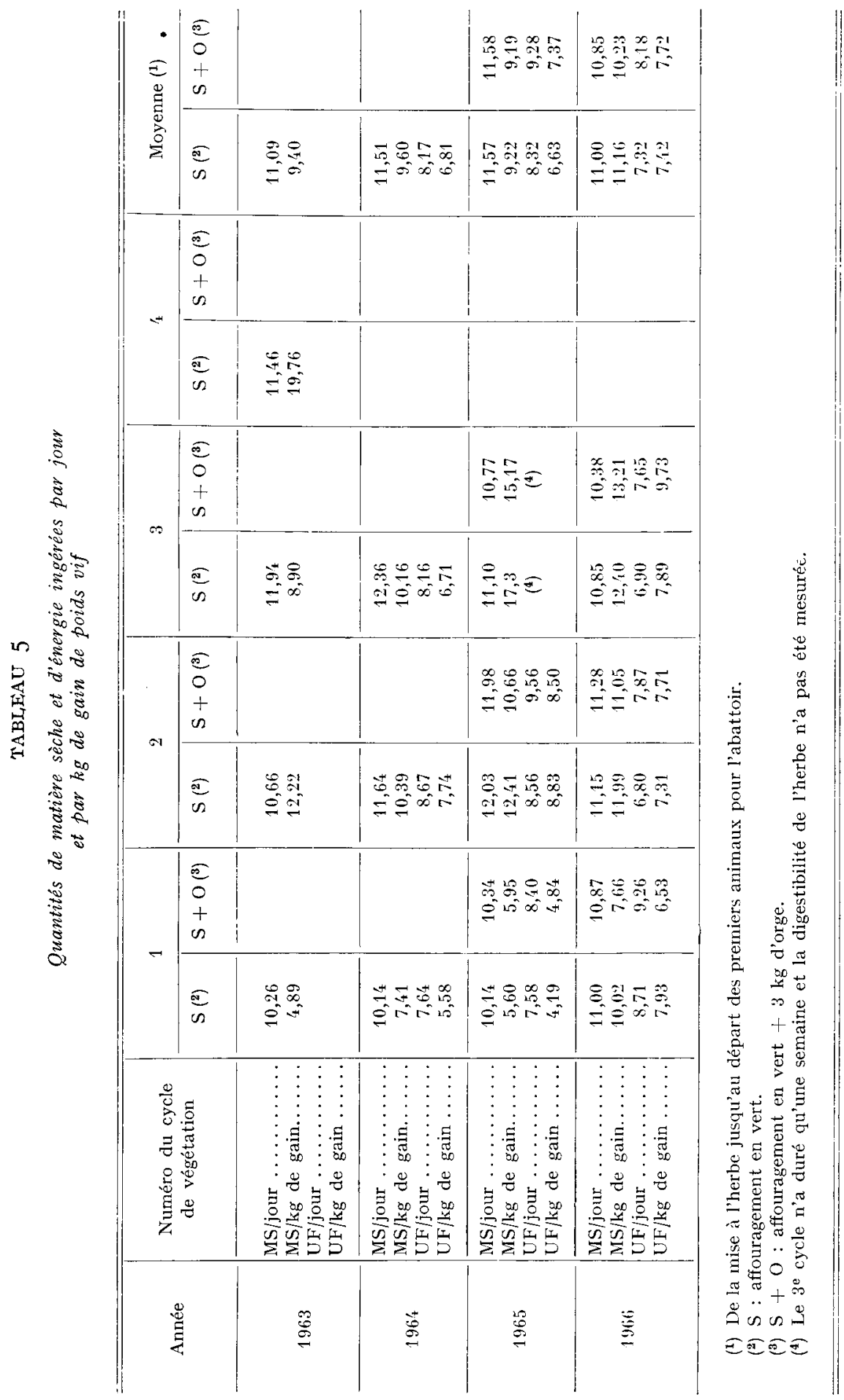
ingérées ont été en moyenne identiques : respectivement 2,21 et $2,23 \mathrm{~kg}$ par Ioo $\mathrm{kg}$ de poids vif. Les $3^{\mathrm{e}}$ cycles de Ray-grass ont cependant été ingérés en plus grande quantité que les $3^{\mathbf{e}}$ cycles de Fétuque des prés (tabl. 4).

D'une façon générale, les animaux recevant $3 \mathrm{~kg}$ d'orge ont consommé la même quantité de matière sèche totale que les animaux ne recevant que le fourrage vert (fig. 5). L'orge s'est donc substituée à l'herbe à poids égal de matière sèche et cela quelle que soit la nature du fourrage distribué (Ray-grass, Fétuque), et le numéro du cycle de végétation.

\section{Quantité d'éléments nutritifs ingérés par les animaux en stabulation.}

La quantité de matière sèche d'herbe ingérée par $\mathrm{kg}$ de gain de poids vif a été en moyenne de $9,85 \mathrm{~kg}$; elle a été peu variable avec l'année $(9,20$ à I I, I5 kg) mais a augmenté de façon très nette entre le premier cycle et les repousses : 7,0 kg contre r I,9 $\mathrm{kg}$ en moyenne (tabl. 5).

La quantité journalière d'énergie nette (estimée à partir de la digestibilité de l'herbe) ingérée par les animaux recevant de l'herbe en stabulation a été en moyenne de 7,94 UF ; elle a relativement peu varié $(6,80$ à $8,7 \mathrm{I})$ avec le numéro du cycle ou l'année. L'ingestion de $3 \mathrm{~kg}$ d'orge en supplément ne l'a augmenté que de o,9I UF/jour (tab1. 5), car l'orge s'est substituée à de l'herbe de bonne digestibilité.

La quantité d'énergie ingérée par $\mathrm{kg}$ de gain de poids vif a été en moyenne de 7,o UF pour les animaux ne recevant que de l'herbe; elle a été peu variable avec l'année $(6,6$ à $7,4 \mathrm{UF})$ mais a été plus élevée pour les repousses ( 7, I 5 en moyenne) que pour le premier cycle $(5,90)$, ce qui résulte d'un gain journalier plus faible et probablement d'une augmentation de la proportion des dépôts adipeux.

Malgré une vitesse de croissance un peu supérieure les animaux recevant de l'orge ont ingéré en moyenne plus d'énergie $(7,5$ contre 7,0 UF) par $\mathrm{kg}$ de gain de poids vif que les animaux ne recevant que de l'herbe, ce qui est en accord avec le fait qu'ils se sont engraissés plus rapidement.

\section{DISCUSSION}

Comparaison des deux systèmes d'affouragement

\section{Croît individuel et engraissement.}

Nos résultats montrent que des bœufs de 24 mois recevant à l'auge de l'herbe fauchée sur des prairies temporaires à base de graminées effectuent des croîts comparables à ceux des bœufs au pâturage et s'engraissent aussi bien et presque aussi vite. L'affouragement en vert semble donc plus favorable à l'engraissement ou à la croissance qu'à la production laitière. En effet, toutes les comparaisons réalisées avec des vaches laitières (cf. revues de Hufman, I959; Arnon, I960; WATSON et Runcie, I 960) mettent en évidence une production laitière individuelle des vaches en stabulation systématiquement un peu inférieure à celle des vaches au pâturage. En revanche, tous les essais réalisés à l'Université de Davis en Californie (ITTNER et al., I954; MEYer et al., I956 ; Hur,L et al., I957 et I96I) montrent que les jeunes bœufs réalisent les mêmes croissances au pâturage et en affouragement en vert. 
La comparaison des deux systèmes d'affouragement impose l'exploitation d'une herbe de même âge, et une alimentation à volonté des animaux. Alors qu'il est facile d'ajuster la superficie à faucher de manière à ce que les animaux en stabulation consomment à volonté, il est plus délicat d'ajuster le chargement des parcelles pâturées à la quantité d'herbe présente. On peut cependant penser que sur les quatre années l'erreur commise a été faible et toujours du même ordre car toute l'expérience a été suivie par le même technicien qui possède une grande habitude des expérimentations de pâturage et qui corrigeait en permanence la superficie des parcelles allouée aux animat1x au pâturage. Cette erreur est d'ailleurs commune à tous les essais aut pâturage.

Les quantités de matière sèche d'herbe ingérées par les bœufs: en moyenne 2,2 I $\mathrm{kg}$ pour Ioo $\mathrm{kg}$ de poids vif avec des extrêmes allant de I,6 à $2,9 \mathrm{~kg}$, sont très voisines de celles que nous avions mesurées sur des vaches laitières à Jouy et à La Minière (DEMARQUILI, I I 66) ; en moyenne $2,22 \mathrm{~kg}$ avec des extrêmes de I, 7 à $2,8 \mathrm{~kg}$. En revanche, la quantité de matière sèche d'herbe ingérée par le bœuf diminue de I $\mathrm{kg}$ par $\mathrm{kg}$ de matière sèche d'aliment concentré ingéré. Cette diminution est plus élevée que celle enregistrée chez la vache : o à $0,6 \mathrm{~kg}$ suivant les auteurs (CorBETT et BOyNe, I950 ; HaNCOCK, I958 ; Holmes et MCLUSKY, I955 ; Holmes, I965).

\section{Utilisation de la prairie.}

Dans nos essais, l'affouragement en vert a permis d'augmenter en moyenne le chargement à l'hectare de 4,0 ou I3,o p. Ioo suivant que l'on considère la période pendant laquelle tous les animaux sont encore présents ou la durée totale de la période d'engraissement. Ces valeurs sont très proches de celle $(8,0)$ trouvée par Hoon (Ig62) avec des bœufs en Grande-Bretagne mais est plus faible que celles trouvées par les auteurs américains, notamment ceux de l'Université de Californie. Il est cependant difficile de comparer les différences de chargements observées suivant les auteurs entre 1'affouragement en vert et le pâturage. En effet, ces différences dépendent d'une part de la nature du fourrage utilisé, comme l'ont bien montré GuI_LICKson et Wirxoc (1956), et les valeurs observées sont tout à fait différentes suivant que les fourrages utilisés sont à port dressé (plantes mieux adaptées à la fauche) ou à port étalé (plantes moins bien adaptées à la fauche). Elles dépendent d'autre part de la proportion de refus tolérée à l'auge (Io à I 5 p. Ioo dans nos essais mais seulement I,7 p. Ioo dans ceux de Hoon (I962)), mais surtout au pâturage, car, dans ce dernier cas, les refus peuvent être très variables et leur importance est le plus souvent inconnue car ils sont appréciés de façon subjective. Cela revient à dire que les différences de chargement entre le pâturage et l'affouragement en vert vont dépendre en grande partie du chargement adopté au pâturage. Ce dernier point soulève d'ailleurs un problème beaucoup plus général puisque les comparaisons de production animale à l'unité de surface entre traitements ne sont valables que si dans chaque traitement, on a adopté le chargement maximum compatible avec les performances animales individuelles désirées comme l'ont souligné Ivrvs et al. (I958).

Malgré un chargement à 1'hectare plus élevé en stabulation, les surfaces fourragères à prévoir sont plus importantes si on veut à certaines périođes ne pas manquer d'herbe, ce qui avait été déjà mis en évidence quand l'herbe est courte par DoNKSER (I958) (cité par ARNon, I960) ; 1'affouragement en vert ne se traduit donc pas par une 
économie de surface mais par des excédents d'herbe plus importants à certains cycles et notamment au $I^{\text {er }}$ cycle, excédents qui peuvent être fauchés et conservés pour 1'hiver.

Bien que le chargement à l'hectare en affouragement en vert ne soit que légèrement supérieur à celui du pâturage, et que les croissances journalières soient identiques au pâturage et en affouragement en vert, les gains de poids vif à l'hectare en affouragement en vert sont supérieurs de II,8 p. Ioo en moyenne. La valeur la plus faible (2,o p. IOO) a été obtenue en I 965 où les prairies utilisées étaient essentiellement à base de Fétuque des prés. Elle est voisine de celle observée (5,0 p. roo) par HooD (I962) également sur de la Fétuque des prés. Cette plante est en effet certainement moins bien adaptée à la fauche que le Ray-grass d'Italie. Cette augmentation de la production animale à l'hectare en affouragement en vert résulte essentiellement du premier cycle de végétation $(37,5 \mathrm{p}$. I00). En revanche, le gain de poids vif à l'hectare a été en moyenne plus élevé $(+9,7$ p. Ioo) au pâturage qu'en stabulation pour les cycles de repousses. Il semble donc que les animaux au pâturage exploitent moins bien les premiers cycles et mieux les repousses que les animaux en stabulation. Indépendamment des causes directement liées aux différences de gain de poids vif entre animaux suivant les traitements qui ont déjà été discutées, cela peut résulter - soit du fait que les refus au pâturage ont été plus élevés au cours des premiers cycles qu'au cours des repousses car dans les conditions du Pin les premiers cycles ont dû être exploités jusqu'à un stade avancé (en outre les repousses ont été très bien exploitées puisque la majorité des deuxièmes cycles pâturés avaient été fauchés au premier cycle) - soit du fait que le chargement au pâturage a été plus faible au premier cycle qu'aux cycles suivants compte tenu de la quantité d'herbe présente.

\section{Possibilités de généralisation et d'application.}

Il faut se garder de généraliser sans vérification préalable nos résultats à des animaux plus jeunes ou recevant des fourrages de nature différente ; c'est ainsi qu'au domaine de la Minière (Yvelines), il nous a été impossible d'engraisser des boufs Normands de 30 mois en leur distribuant à l'auge de la luzerne verte même avec une complémentation de $3 \mathrm{~kg}$ d'orge par animal et par jour.

En outre, la comparaison du pâturage et de l'affouragement en vert a été conduite d'une façon bien définie qui mérite d'être soulignée. Dans tous les essais, 1'exploitation de l'herbe a été menée de façon parallèle dans les deux traitements et les animaux ont toujours consommé une herbe de même nature et de même âge. Il aurait été intéressant d'introduire dans ces essais un deuxième lot qui aurait été conduit de façon indépendante avec un rythme d'exploitation plus rapide. En effet, il a fallu au début de chaque cycle attendre que l'herbe soit suffisamment haute pour être fauchée alors qu'il aurait été possible de commencer l'exploitation par le pâturage. Avec un rythme d'exploitation plus rapide, les croissances au pâturage auraient été peut-être un peu plus élevées. En revanche, les refus que nous avons tolérés en stabulation sont beaucoup plus élevés que ce qu'on pourrait tolérer dans la pratique (au détriment de l'état d'engraissement) et la production animale à l'hectare fauché est donc sous-estimée. En outre, comme nous avons été amenés à changer de parcelles à l'intérieur d'un même cycle de végétation, il est difficile, à partir de nos résultats de mettre en évidence : 
- I'influence éventuelle de la nature de l'herbe sur les résultats comparatifs obtenus par le pâturage et par l'affouragement en vert,

- l'effet cumulatif d'une pâture ou d'une fauche à chaque cycle végétatif sur la production (végétale ou animale) globale annuelle d'une prairie.

La distribution de $3 \mathrm{~kg}$ d'orge par jour en supplément de la ration d'herbe verte permet de diminuer la période d'engraissement et de réduire la surface fourragère nécessaire, ce qui peut être intéressant daus les zones céréalières. Cette distribution d'orge pourrait peut-être être limitée et ne commencer qu'au deuxième ou au troisième cycle quand la production des prairies diminue. Elle permet alors une nette économie de surface, en moyenne 23,0 p. Ioo dans nos essais.

D'après les quelques mesures de temps de travaux que nous avons effectuées, il ressort que l'affouragement en vert demande 4 fois plus d'heures de main-d'œuvre que le pâturage. Ces chiffres sont évidemment plus importants que ceux trouvés avec des vaches laitières où les rapports sont à peine de I à 2 (BATEMAx́ et al., I959) à cause de la traite qui est facilitée chez les vaches en stabulation.

En outre, l'affouragement en vert suppose un investissement en matériel de récolte et de distribution important bien que ce matériel soit assez polyvalent et puisse servir à la récolte des fourrages conservés. Le problème est donc de savoir si le supplément de production animale à l'hectare paiera les frais supplémentaires de main-d'œuvre et d'investissement. Ce calcul doit être laissé aux économistes. Il ne faut cependant pas oublier que l'intérêt de l'affouragement en vert réside peut-être essentiellement dans la possibilité d'utiliser à partir du mois de juillet, en supplément ou en remplacement de l'herbe, des fourrages beaucoup plus productifs (luzerne, sorgho, maïs) que les plantes prairiales, fourrages qui s'exploitent très difficilement en pâturage. Il permet en outre d'utiliser des parcelles non clôturées et sans point d'eau. En revanche, l'affouragement en vert soulève de très gros problèmes de récolte dans certains types de sol lors des périodes pluvieuses prolongées. C'était notamment le cas chaque printemps, au domaine du Pin-au-Haras.

Reçu pour publication en septembre 1969.

\section{SUMMARY}

\section{COMPARISON OF GRAZING VERSUS GREEN CHOP FEEDING FOR BEEF CATTIE PRODUCTION}

I. At the Pin aux Haras experimental farm in Normandy, we compared the growth and fattening ratesof 2 lots of 30 -month old beef cattle for 4 successive years from 1963 to 1966 . These cattle grazed grass leys, or were given grass of the same age in the stable, mowed in meadows in the parts reserved for cutting. In I 965 and I966, a third lot of stabled animals received $3 \mathrm{~kg}$ of barley per day in addition to fed ad libitum grass. Table I shows distribution by breed and the initial weight of the animals according to years and trials.

2. Grass leys were used having a single grass specie (perennial or Italian rye-grass, meadow fescue) associated with white clover. Figure I shows the speciss employed and the exploitation calendars. The beef cattle at pasture ate the grass of those meadows in rationed grazing, the electric fences before and behind being moved forward every 4 days. The grass necessary for the stabled animals was cut every morning with a classic mower, and picked up by a self-loading trailor. It was fed ad libitum (io to $15 \mathrm{p}$. Ioo refused) twice a day-- at 8 a.m. and 2 p.m., and the refusals were removed once a day. 
3. When we commenced giving grass, stabled animals were weighed every days, and those at pasture every 2 days up to the time when their weight ceased to decrease. The lowest average daily weight recorded was chosen as the initial lot weight. The animals were then weighed at the end of each vegetation cycle, and again when they went to slaughter. Animals were slaughtered when they were sufficiently fattened. The quantities of green matter and dry matter ingested per animal in each stabled lot of groups of 5 or 6 , were measured every day except Sunday. Beginning in 1964 , the digestibility of the grass distributed to those animals was measured on a lot of 4 wethers.

The climatic conditions during these trials are given in figure 2.

4. The digestibility of forages used from 1964 to 1966 is shown in figure 3 . On the whole, it was good (about $70 \mathrm{p}$. I oo) and remained between 65 and $75 \mathrm{p}$. 100, except at the beginning of the first cycles, and for som second growth herbage containing flowering hearls.

5. Live weight gains (table 2) were computed in two ways :

- for the period during which all the animals were in experiment; the lots were thoroughly compared;

- for the entire experimental period, including the time during which some already fattened animals left for the slaughter-house.

Average live weight gain of stabled and grazing animals, respectively, for the four years was I. I.55 g and I.I $26 \mathrm{~g}$ per day from the time grass was given until the first animals were slaughtered (beginning of September), and $\mathrm{r} 039$ and $\mathrm{I} 067 \mathrm{~g}$ per day for the total fattening period. These values are not significantly different. Compared to grazing animals, stabled animals fattened significantly more during the first vegetation cycle (I $594 \mathrm{~g} /$ day against I $305 \mathrm{~g} / \mathrm{day}$ ), but less during the regrowth cycles-- $973 \mathrm{~g} / \mathrm{day}$ against I I 8 g/day (significantly different) in the second cycle, I $030 \mathrm{~g}$ against I $108 \mathrm{~g} /$ day (insignificantly different) during the third cycle

Individual live weight gains increased relatively little (about $40 \mathrm{~g} /$ day up to the beginning. September, and about $\mathrm{I} 7 \mathrm{O} \mathrm{g} /$ day for the total fattening period) when stabled animals received $3 \mathrm{~kg}$ of barley por day. Gain was only significantly increased in I966, and then only when computed for the total fattening period. On the other hand, feeding barley significantly shortened the fattening pariod by about 20 days (I 20 against 140 days).

6. The average amount of live weight gain per hectare was $75 \mathrm{I} \mathrm{kg}$ per mowed hectare as against $672 \mathrm{~kg}$ per pasture hectare, or a difference of I I.8 p. Ioo. With the apport of $3 \mathrm{~kg}$ of barley, there was an average increase of 36.3 p. Ioo live weight gain for one mowed hectare (table 3 ).

7. The dry matter intake varied from 1.57 to $2.90 \mathrm{~kg}$ per $\mathrm{I} 00 \mathrm{~kg}$ of live weight (fig. 3), and averaged $2.2 \mathrm{I} \mathrm{kg}$. It was independent of digestibility, the dry matter content, and the type (ryegrass or meadow fescue) of grass distributed (table 4 ). The intake varied more at the beginning and the end of the trials, and tended to diminish with the number of the vegetation cycle (fig. 4, table 4 ). Animals receiving $3 \mathrm{~kg}$ of barley ingested the same quantity of total dry matter as animals given only grass (fig. 5). Barley may therefore be substituted for grass by equal dry weight.

8. The grass intake ingested per $\mathrm{kg}$ of liveweight gain averaged $9.85 \mathrm{~kg}$ of dry matter. It increased markedly between the first cycle $(7.0 \mathrm{~kg})$ and the regrowths (II.9 $\mathrm{kg})$ (table 5). The amount of net energy (estimated by grass digestibility and expressed in feed units--U.F.) ingested per $\mathrm{kg}$ of gain averaged $7.0 \mathrm{U}$.F. for animals receiving only grass, and $7.5 \mathrm{U}$.F. for animals receiving a supplement of $3 \mathrm{~kg}$ of barley. This supports the fact that they fattened more rapidly.

9. These results show that 24 -month old beef cattle receiving grass in the feed trough cut from grass leys, grow comparably as well as cattle at pasture, and fatten as well and as quickly. On the other hand, feeding fresh green roughage produces only a small increase in live weight gain per hectare. Its economic interest may lie in the possibility of using more productive forages (alfalfa-sorghum-corn) than meadow plants beginning in July.

\section{RÉFÉRENCES BIBLIOGRAPHIQUES}

Arxox I., I960. Grazing versus feeding cut forage crops. Proc. 8 th Internat. Grassl. Congr., 648-652. Batemax G. Q., Stoddard G. E., Nidielson G. H., 1958. Self service or maid service. Should a dairy cow go and get it or have it brought to her? Utah Agric. Exp. Sta., Farm and Home Service, I9-2.

Corbett J. C., Borne A. W., 1958. The effects of a low-protein food supplement on the yield and composition of milk from grazing dairy cows and on the composition of their diet. J. Agric., Sci., 41, 95-107. 
Demarqully C., JARRIGE R., I964. Valeur alimentaire de l'herbe des prairies temporaires aux stades d'exploitation pour le pâturage. I. Composition chimique et digestibilité. Ann. Zootech., 13, 3 or-339.

Gullickson T. W., Wilcox C. L., I956. Silage or rotational grazing. Minnesota Farm and Home Sci., 13, I 8-19.

Hancock J., I958. The conversion of pasture to milk. The effect of stocking rate and concentrate feeding. J. Agric. Sci., 50, 284-296.

Holmes W., Jones J. G. W., Drake-Brockman R. M., White N., rg65. The feed intake of milk cows. I. Intake of winter rations during pregnancy and lactation and the influence of change to pasture. Animal Prod., 7, 27-37.

Holmes W., Mc Lusky D. S., 1955. In "Animal production from grass". (Europ Assoc. Anim. Prod. Public. ne 6), p. 29.

Hoop A. E. M., I962. Soilage for beef cattle. J. brit. Grassl. Soc., 17, 264-267.

Huffman C. F., 1959. Summer feeding of dairy cattle. A review. J. Dairy Sci., 42, 1495-1551.

Hull J. L., Meyer J. H., Lofgreen G. P., Strother A., r957. Studies on forage utilization by steers and sheep. J. anim. Sci, 16, 757-765.

Hull J. L., MeYer J. H., Kromann R., I96r. Influence of stocking rate on animal and forage production from irrigated pasture. J. anim. Sci, 20, 46-52.

ItTNer N. R., Lofgreen G. P., Meyer J. H., I954. A study of pasturing and soiling alfalfa with beef steers. J. anim. Sci., 13, $37 \cdot 43$.

Ivins J. D., Dilnot J., Davidson J., 1958. The interpretation of data of grassland evaluation in relation to the varying potential outputs of grassland and livestock, J. brit. Grassl. Soc., 13, 23.

MeYer J. H., LOFGreen G. P., ItTNER N. R., 1956. Further studies in the utilization of alfalfa by beef steers. J. anim. Sci, 15, 64-67.

Watson S. J., Runcie K. V., 1960. Soiling or zero-grazing. Outlook on Agriculture, 2, $264-275$. 